\title{
PRODUÇÃO DE HIDROLASES POR FERMENTAÇÃO SÓLIDA UTILIZÁNDO RESÍDUOS DA HIDRODESTILAÇÃO DE ESPÉCIES DO GÊNERO Aniba
}

\author{
P. M. ALBUQUERQUE ${ }^{1}$, A. C. FISCHBORN ${ }^{1}$, L. E. S. BARATA ${ }^{2}$ e S. DUVOISIN Jr ${ }^{3}$ \\ ${ }^{1}$ Universidade do Estado do Amazonas, PPG em Biotecnologia e Recursos Naturais da Amazônia \\ ${ }^{2}$ Universidade Federal do Oeste do Pará, PPG em Recursos Naturais da Amazônia \\ ${ }^{3}$ Universidade do Estado do Amazonas, Escola Superior de Tecnologia, Engenharia Química \\ E-mail para contato: palbuquerque @uea.edu.br
}

\begin{abstract}
RESUMO - A indústria de óleos essenciais gera resíduos que podem ser reaproveitados como substratos em processos biotecnológicos. Neste trabalho o resíduo sólido gerado após a extração do óleo essencial de Aniba parviflora e Aniba rosaeodora foi utilizado na fermentação sólida do fungo Trichoderma harzianum, para obtenção de celulase e xilanase, enzimas com ampla aplicação industrial. Foi realizado um planejamento experimental fracionado para selecionar as variáveis (umidade do substrato, tempo, temperatura e adição dos nutrientes nitrogênio e fósforo) que influenciam de forma significativa as atividades enzimáticas, utilizando o fungo inoculado na forma de disco micelial e suspensão de esporos. O fungo mostrou maior produção de celulase $(2,71$ U/gbs) em níveis máximos de tempo, temperatura e umidade, e níveis mínimos de nitrogênio e fósforo. A umidade foi a variável estatisticamente significativa para a produção das duas enzimas. O resíduo de macacaporanga mostrou-se mais adequado para o cultivo fúngico.
\end{abstract}

\section{INTRODUÇÃO}

Espécies do gênero Aniba, pertencente à família Lauraceae são conhecidas pela produção e constituição dos seus óleos essenciais, utilizados na indústria de perfumaria, destacando-se a espécie Aniba rosaeadora (pau-rosa), que possui o linalol como principal constituinte do seu óleo essencial, muito utilizado como fixador (Melo et al., 2006). Outra espécie, a A. parviflora (Meissn) Mez (macacaporanga) também vem sendo utilizada na perfumaria (Marques, 2001).

Técnicas de extração dos compostos aromáticos de plantas, incluindo os óleos essenciais e os extratos, são citadas na literatura, entre elas a extração com solventes orgânicos, enfloração, prensagem, utilização de $\mathrm{CO}_{2}$ supercrítico, hidrodestilação por arraste a vapor (Pybus e Sell, 1999). Nesta última, além de se obter resíduo líquido, conhecido como hidrolato, também se obtêm resíduos sólidos, os quais podem ser utilizados como substratos em bioprocessos.

Os processos biotecnológicos têm conquistado um lugar de destaque no desenvolvimento tecnológico mundial, exibindo características econômicas e operacionais que conferem vantagens em 


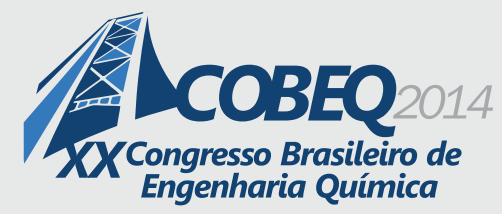

19 a 22 de outubro de 2014

Florianópolis/SC

relação aos processos químicos convencionais. O uso desses processos possibilita a produção de um grande número de metabólitos de interesse industrial, incluindo enzimas, as quais podem ser obtidas a partir do reaproveitamento de resíduos da agroindústria que podem ser encontrados em abundância no Brasil, contribuindo assim, para a redução de problemas ambientais (Maciel, 2006).

Muitas são as fontes de enzimas utilizadas industrialmente. Embora algumas sejam extraídas de tecidos animais e vegetais, as enzimas usadas na indústria são geralmente obtidas de microrganismos. A grande variedade e disponibilidade de bactérias, leveduras e fungos, aliada ao domínio das técnicas de cultivo, tornam as enzimas de origem microbiana as mais utilizadas em processos industriais. Apesar de um microrganismo ser capaz de produzir mais de mil enzimas distintas, é necessário um trabalho cuidadoso para o isolamento de espécies que produzam determinadas enzimas com as características desejadas (Faber, 2004).

Os ambientes tropicais brasileiros mostram uma rica biodiversidade, tornando-se promissores para a pesquisa de novos microrganismos com aplicações biotecnológicas, em especial os fungos. Numerosos fungos, como os dos gêneros Trichoderma, Aspergillus e Penicillium são capazes de produzir celulases e xilanases em diferentes condições (Souza et al., 2009). Diversos estudos têm reportado a produção de enzimas utilizando a Fermentação Sólida (FS), processo onde o crescimento microbiano e a formação de produtos ocorrem na superfície de substratos sólidos (Souza et al., 2009). A FS é uma excelente alternativa para a utilização de resíduos agroindustriais (Singhania et al., 2009).

As xilanases são um grupo de enzimas presente nas mais diversas aplicações industriais, destacando-se especialmente na indústria de polpa e papel, com significativa expansão de aplicações no setor de alimentos e em rações animais. São enzimas que degradam a xilana, a qual é o principal componente da hemicelulose vegetal (Metzler, 2001). A celulase também é uma enzima de grande interesse. O nome celulase corresponde às diversas enzimas presentes na biodegradação da celulose. Sua aplicação industrial atinge áreas como a produção de bebidas, alimentos, rações, indústria têxtil, bem como a hidrólise enzimática da celulose, com objetivo de se obter açúcares fermentáveis, utilizados para produção de biocombustíveis (Mussatto et al., 2007).

Portanto, o objetivo deste trabalho, foi investigar, por meio de um planejamento experimental, a produção de hidrolases fúngicas a partir do aproveitamento do resíduo da obtenção do óleo essencial de duas espécies de Lauráceas, visando a utilização sustentável de plantios florestais que possibilite o desenvolvimento de tecnologias adequadas à realidade da Região Amazônica.

\section{MATERIAIS E MÉTODOS}

\subsection{Resíduo Sólido}

O resíduo sólido utilizado como substrato na fermentação foi obtido da hidrodestilação dos galhos finos ( $\pm 3 \mathrm{~cm}$ de diâmetro) e folhas das espécies A. parviflora e A. rosaeodora, coletadas na Fazenda Pematec Santarém-PA e na Fazenda Magaldi (Maués-AM), respectivamente. O material vegetal foi seco à temperatura ambiente durante 10 dias e triturado em moinho de facas com tela de 3 mm, sendo utilizado para a extração do óleo essencial em hidrodestilador tipo Clevenger. 


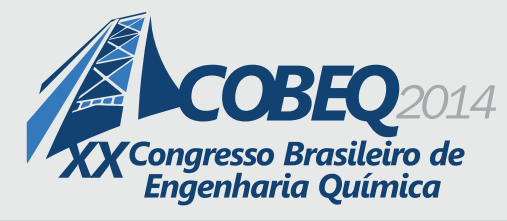

A umidade do resíduo da $A$. parviflora e da $A$. rosaeodora foi obtida pelo método de dessecação, com o aquecimento direto da amostra a $50^{\circ} \mathrm{C}$ em estufa, de acordo com as Normas Analíticas do Instituto Adolfo Lutz (1985). A partir do cálculo da umidade foi determinada a quantidade de solução nutriente que foi adicionada nos experimentos de FS.

\subsection{Microrganismo}

Foi utilizada a espécie fúngica Trichoderma harzianum (DPUA 188/UFAM), cedido pelo Laboratório de Micologia da Universidade Federal do Amazonas (UFAM). O fungo foi mantido em meio ágar-aveia e repicado periodicamente. Para o preparo do inóculo, o fungo foi cultivado utilizando o mesmo meio de cultura, sendo incubado em estufa BOD durante sete dias a $28^{\circ} \mathrm{C}$.

Duas formas de inóculo fúngico, disco micelial e suspensão de esporos, foram utilizadas para avaliar a produção das enzimas celulase e xilanase por meio da FS. Das placas cultivadas com o fungo $T$. harzianum foram cortados discos miceliais de $1,0 \mathrm{~cm}$ de diâmetro, os quais foram retirados e inoculados no meio sólido para a produção de enzimas. A suspensão de esporos foi preparada a partir das placas cultivadas com o fungo esporulado, preparando-se uma solução salina a $1 \%$, sendo padronizado na concentração de $1 \times 10^{8} \mathrm{UFC} / \mathrm{mL}$.

\subsection{Cultivo dos Fungos Utilizando a Fermentação Sólida}

Os fungos foram cultivados no resíduo sólido complementado com uma solução de nutrientes. $\mathrm{O}$ meio de cultivo foi composto por $7 \mathrm{~g}$ de resíduo sólido seco e triturado acrescidos de solução contendo diferentes concentrações dos nutrientes $\left(\mathrm{NH}_{4} \mathrm{NO}_{3}\right.$ e $\left.\mathrm{KH}_{2} \mathrm{PO}_{4}\right)$, com quantidade de água deionizada suficiente para atingir os valores de umidade estudados, sendo posteriormente autoclavado. Em seguida, cinco discos miceliais ou $50 \mu \mathrm{L}$ da suspensão de esporos foram inoculados no meio de cultivo sólido mantido a diferentes temperaturas e períodos de tempo.

Após o crescimento, $20 \mathrm{~mL}$ de água deionizada autoclavada foram adicionadas ao meio, para facilitar a extração das enzimas. O meio contendo água foi colocado em um banho com ultra-som por 2 min e sob em shaker por 10 min. Foi realizada uma filtração a vácuo, em funil de Büchner e as amostras dos extratos enzimáticos foram armazenadas em freezer.

\subsection{Avaliação das Atividades Enzimáticas}

A partir da metodologia de Silva e Carmona (2008), a atividade de enzimas celulolíticas foi determinada pela avaliação da atividade de endoglucanase (carboximetilcelulase), que consistiu na adição de $500 \mu \mathrm{L}$ do extrato bruto a $500 \mu \mathrm{L}$ do substrato CMC a $1 \%$ em tampão acetato de sódio 50

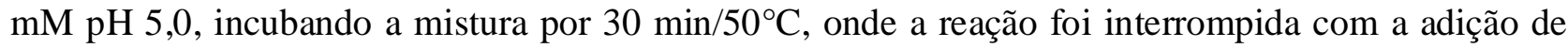
$500 \mu \mathrm{L}$ de ácido 3,5-dinitrosalicílico (DNS). Após, agitou-se no vórtex e manteve-se por 5 $\mathrm{min} / 100^{\circ} \mathrm{C}$. Resfriou-se em temperatura ambiente e, em seguida, adicionou-se água destilada, completando para $10 \mathrm{~mL}$. A leitura foi feita em espectrofotômetro a $540 \mathrm{~nm}$.

Para a atividade xilanolítica também seguiu-se a metodologia de Silva e Carmona (2008), onde 


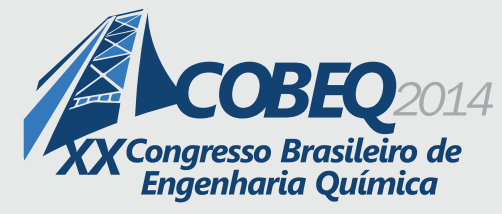

a mesma foi determinada por meio de incubação de $500 \mu \mathrm{L}$ do extrato enzimático com $750 \mu \mathrm{L}$ de solução de xilano a $1 \%$ em tampão fosfato $50 \mathrm{mM} \mathrm{pH} 6,0$ por $10 \mathrm{~min} / 50^{\circ} \mathrm{C}$. Adicionou-se $500 \mu \mathrm{L}$ de DNS e incubou-se a $100^{\circ} \mathrm{C} / 5 \mathrm{~min}$. Em seguida, foram adicionados $8.250 \mu \mathrm{L}$ de água destilada e as soluções foram homogeneizadas. Por fim, foi feita a leitura no espectrofotômetro a $540 \mathrm{~nm}$.

O cálculo da atividade enzimática foi realizado a partir da determinação dos açúcares redutores liberados em cada reação conforme a metodologia descrita por Miller (1959). Uma unidade de atividade enzimática foi definida como aquela correspondente à liberação de um $\mu$ mol de açúcar redutor equivalente à glicose ou xilose, por minuto, por $\mathrm{mL}$ da amostra, nas condições do ensaio. Após a obtenção da atividade em $\mathrm{U} / \mathrm{mL}$, usando os valores de umidade de cada experimento, converteram-se os valores para U/g de substrato (em base seca).

\subsection{Planejamento Experimental}

Foi realizado um planejamento experimental fracionado $2^{5-1}$ para selecionar as variáveis umidade do substrato, adição de nutrientes (fontes de nitrogênio e de fósforo), tempo e temperatura de cultivo - que influenciam de forma estatisticamente significativa as atividades enzimáticas $(\mathrm{p}<0,05)$.

O software Statistica 10.0 foi utilizado para analisar os dados experimentais. Assim, utilizando a ferramenta do planejamento experimental foi possível investigar a influência das variáveis na produção enzimática. O erro experimental foi avaliado a partir da triplicata no ponto central (Rodrigues e Iemma, 2005).

\section{RESULTADOS E DISCUSSÃO}

Os resultados da atividade enzimática celulolítica (AEC) e xilanolítica (AEX) obtidos a partir do planejamento experimental fatorial fracionado $2^{5-1}$ com o cultivo do fungo T. harzianum em FS, para cada tipo de inóculo utilizado (DM e SE) e para os dois substratos sólidos (resíduos de macacaporanga e de pau-rosa), estão apresentados na Tabela 1.

Para o resíduo de macacaporanga, observa-se que o melhor resultado para a atividade de celulase $(2,71 \mathrm{U} / \mathrm{gbs}=0,45 \mathrm{U} / \mathrm{mL})$ foi obtido no ensaio 4 , quando utilizado como inóculo a SE, níveis máximos de umidade $(90 \%)$, temperatura $\left(30^{\circ} \mathrm{C}\right)$ e tempo de cultivo $(15$ dias $)$ e níveis mínimos de fósforo $(3,0 \%)$ e nitrogênio $(1,5 \%)$. Já para o resíduo de pau-rosa, o melhor resultado $(1,59 \mathrm{U} / \mathrm{gbs}=$ $0,25 \mathrm{U} / \mathrm{mL}$ ) foi obtido no ensaio 11 , quando utilizado como inóculo o $\mathrm{DM}$, níveis máximos de umidade $(90 \%)$, tempo de cultivo (15 dias) e fósforo $(6,0 \%)$ e níveis mínimos de temperatura $\left(24^{\circ} \mathrm{C}\right)$ e nitrogênio $(1,5 \%)$.

Para o resíduo de macacaporanga, observa-se que o melhor resultado para a atividade de xilanase $(1,14 \mathrm{U} / \mathrm{gbs}=0,15 \mathrm{U} / \mathrm{mL})$ foi obtido no ensaio 6 , quando utilizado como inóculo a SE, níveis máximos de umidade $(90 \%)$, temperatura $\left(30^{\circ} \mathrm{C}\right)$ e nitrogênio $(4,5 \%)$ e níveis mínimos do tempo de cultivo (9 dias) e de fósforo (3,0\%). Já para o resíduo de pau-rosa, o melhor resultado $(0,92 \mathrm{U} / \mathrm{gbs}=$ $0,14 \mathrm{U} / \mathrm{mL}$ ) foi obtido no ensaio 16 , quando utilizado como inóculo o $\mathrm{DM}$, níveis máximos de umidade $(90 \%)$, tempo de cultivo (15 dias), temperatura $\left(30^{\circ} \mathrm{C}\right)$, fósforo $(6,0 \%)$ e nitrogênio $(4,5 \%)$. 


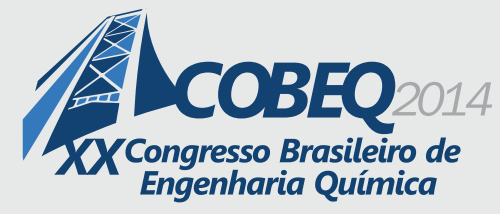

Tabela 1 - Atividades enzimáticas de celulase e xilanase obtidas nos ensaios de fermentação sólida.

\begin{tabular}{|c|c|c|c|c|c|c|c|c|c|c|c|c|c|}
\hline \multirow{3}{*}{ ENSAIOS } & \multirow{2}{*}{\multicolumn{5}{|c|}{ VARIÁVEIS }} & \multicolumn{4}{|c|}{ MACACAPORANGA } & \multicolumn{4}{|c|}{ PAU-ROSA } \\
\hline & & & & & & \multicolumn{2}{|c|}{$\mathrm{AEC}(\mathrm{U} / \mathrm{gbs})$} & \multicolumn{2}{|c|}{ AEX (U/gbs) } & \multicolumn{2}{|c|}{$\mathrm{AEC}(\mathrm{U} / \mathrm{gbs})$} & \multicolumn{2}{|c|}{ AEX (U/gbs) } \\
\hline & $\mathrm{T}\left({ }^{\circ} \mathrm{C}\right)$ & $\mathrm{t}$ (dias) & $\mathrm{N}(\%)$ & $\mathrm{P}(\%)$ & $\mathrm{U}(\%)$ & $\mathrm{DM}$ & SE & $\mathrm{DM}$ & $\mathrm{SE}$ & $\mathrm{DM}$ & SE & DM & $\mathrm{SE}$ \\
\hline 1 & $24(-1)$ & $9(-1)$ & $1,5(-1)$ & $3,0(-1)$ & $90(+1)$ & $-0,06$ & $-0,03$ & $-0,92$ & 0,09 & 1,48 & 0,48 & $-2,44$ & 0,86 \\
\hline 2 & $30(+1)$ & $9(-1)$ & $1,5(-1)$ & $3,0(-1)$ & $70(-1)$ & 0,02 & 0,60 & 0,60 & $-0,09$ & 0,58 & 0,02 & $-0,18$ & $-0,41$ \\
\hline 3 & $24(-1)$ & $15(+1)$ & $1,5(-1)$ & $3,0(-1)$ & $70(-1)$ & 0,09 & 0,20 & $-1,35$ & 0,39 & 0,01 & $-0,04$ & 0,10 & 0,09 \\
\hline 4 & $30(+1)$ & $15(+1)$ & $1,5(-1)$ & $3,0(-1)$ & $90(+1)$ & 0,13 & 2,71 & $-0,35$ & 1,01 & 0,15 & 0,20 & 0,26 & 0,20 \\
\hline 5 & $24(-1)$ & $9(-1)$ & $4,5(+1)$ & $3,0(-1)$ & $70(-1)$ & 0,01 & 0,22 & $-0,12$ & $-0,01$ & 0,77 & $-0,01$ & $-0,04$ & 0,01 \\
\hline 6 & $30(+1)$ & $9(-1)$ & $4,5(+1)$ & $3,0(-1)$ & $90(+1)$ & $-0,05$ & 1,31 & 0,75 & 1,14 & 0,57 & 0,25 & $-2,8$ & 0,22 \\
\hline 7 & $24(-1)$ & $15(+1)$ & $4,5(+1)$ & $3,0(-1)$ & $90(+1)$ & 0,21 & 1,13 & 0,09 & 0,88 & 0,14 & 0,46 & $-0,57$ & $-0,18$ \\
\hline 8 & $30(+1)$ & $15(+1)$ & $4,5(+1)$ & $3,0(-1)$ & $70(-1)$ & 0,06 & 0,21 & 0,09 & $-0,09$ & 0,16 & 0,19 & 0,13 & $-0,06$ \\
\hline 9 & $24(-1)$ & $9(-1)$ & $1,5(-1)$ & $6,0(+1)$ & $70(-1)$ & $-0,15$ & 0,23 & $-0,41$ & 0,41 & 0,07 & 0,18 & 0,10 & 0,16 \\
\hline 10 & $30(+1)$ & $9(-1)$ & $1,5(-1)$ & $6,0(+1)$ & $90(+1)$ & $-0,59$ & 0,83 & $-0,92$ & 1,05 & 1,36 & 0,09 & $-1,51$ & 0,39 \\
\hline 11 & $24(-1)$ & $15(+1)$ & $1,5(-1)$ & $6,0(+1)$ & $90(+1)$ & 0,18 & 0,78 & $-0,31$ & 0,61 & 1,59 & 0,14 & $-2,90$ & $-1,54$ \\
\hline 12 & $30(+1)$ & $15(+1)$ & $1,5(-1)$ & $6,0(+1)$ & $70(-1)$ & $-0,03$ & $-0,09$ & $-0,42$ & 0,12 & $-0,01$ & 0,06 & $-0,03$ & $-0,18$ \\
\hline 13 & $24(-1)$ & $9(-1)$ & $4,5(+1)$ & $6,0(+1)$ & $90(+1)$ & 0,09 & 0,42 & 0,39 & 0,26 & 0,93 & 0,14 & 0,83 & 0,22 \\
\hline 14 & $30(+1)$ & $9(-1)$ & $4,5(+1)$ & $6,0(+1)$ & $70(-1)$ & $-0,11$ & 0,21 & 0,26 & $-0,03$ & 0,03 & 0,28 & 0,09 & $-0,07$ \\
\hline 15 & $24(-1)$ & $15(+1)$ & $4,5(+1)$ & $6,0(+1)$ & $70(-1)$ & 0,03 & $-0,09$ & $-0,18$ & 0,15 & 0,19 & 0,05 & $-0,18$ & $-0,03$ \\
\hline 16 & $30(+1)$ & $15(+1)$ & $4,5(+1)$ & $6,0(+1)$ & $90(+1)$ & 0,13 & $-0,01$ & 0,75 & 0,18 & $-0,13$ & 0,64 & 0,92 & 0,26 \\
\hline 17 & $27(0)$ & $12(0)$ & $3(0)$ & $4,5(0)$ & $80(0)$ & 0,22 & 0,11 & $-0,22$ & 0,28 & $-0,48$ & 0,05 & 0,53 & $-0,42$ \\
\hline 18 & $27(0)$ & $12(0)$ & $3(0)$ & $4,5(0)$ & $80(0)$ & 0,27 & $-0,01$ & 0,59 & 0,61 & $-0,62$ & 0,01 & $-1,34$ & 0,07 \\
\hline 19 & $27(0)$ & $12(0)$ & $3(0)$ & $4,5(0)$ & $80(0)$ & 0,54 & 0,11 & $-0,15$ & 0,55 & $-0,55$ & 0,05 & $-0,26$ & 0,02 \\
\hline
\end{tabular}

$\mathrm{T}$ = temperatura; $\mathrm{t}=$ tempo; $\mathrm{N}$ = nitrogênio; $\mathrm{P}$ = fósforo; $\mathrm{U}$ = umidade; $\mathrm{DM}=$ disco micelial; $\mathrm{SE}$ = suspensão de esporos; $\mathrm{AEC}=$ atividade enzimática celulolítica; $\mathrm{AEX}$ = atividade enzimática xilanolítica.

Em geral, os valores de atividades enzimáticas (celulase e xilanase) estão abaixo do esperado. A baixa produção das hidrolases pode ser explicada pelo fato de que as espécies A. parviflora e A. rosaeodora, das quais os resíduos foram utilizados, produzem linalol. Esta substância apresenta efeito antifúngico para alguns fungos, o que pode ter inibido o crescimento do $T$. harzianum, em especial quando o resíduo de pau-rosa foi utilizado, uma vez que a concentração de linalol nesta espécie varia de 74 a 96\% no seu óleo essencial (Maia et al., 2007), enquanto que na macacaporanga, o linalol está presente, em média, na concentração de 35\% (Marques, 2001).

Ao contrário do que foi observado no presente trabalho, Ruegger e Tauk-Tornisielo (2004) utilizaram o fungo $T$. harzianum na produção de CMcase e obtiveram um bom valor de atividade enzimática $(1,64 \mathrm{U} / \mathrm{mL})$ após 4 dias de cultivo a uma temperatura de $25^{\circ} \mathrm{C}$ em farelo de trigo. Quando cultivado no resíduo de macacaporanga, a atividade máxima de CMCase observada foi de $0,45 \mathrm{U} / \mathrm{mL}$ e de $0,25 \mathrm{U} / \mathrm{mL}$ quando o resíduo de pau-rosa foi empregado.

Para a produção de xilanase, esta espécie fúngica também já foi relatada como promissora. Rezende et al. (2002) utilizaram o bagaço de cana-de-açúcar como substrato para a produção de xilanase pelo fungo T. harzianum Rifai, verificando a maior AEX (288 U/mL) no sétimo dia de cultivo. No presente trabalho, o fungo T. harzianum não apresentou resultado satisfatório para a AEX quando cultivado nos resíduos de macacaporanga $(0,15 \mathrm{U} / \mathrm{mL})$ e pau-rosa $(0,14 \mathrm{U} / \mathrm{mL})$.

Em geral, as espécies do gênero Trichoderma são relatadas como boas produtoras de celulases e 


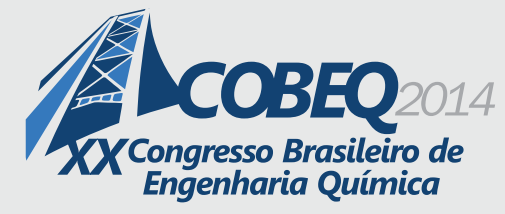

19 a 22 de outubro de 2014

Florianópolis/SC

xilanases, independentemente de suas condições de cultivo. No trabalho de Maurya et al. (2012), por exemplo, foi obtida a produção máxima de celulase $(2,63 \mathrm{U} / \mathrm{mL})$ com o fungo $T$. reesei utilizando como substrato farelo de trigo com umidade de $70 \%$, a $30^{\circ} \mathrm{C}$ após 6 dias de cultivo. Já no trabalho de Kar et al. (2006), a produção máxima de xilanase $(4,75 \mathrm{U} / \mathrm{mL})$ com o fungo $T$. reesei foi obtida em condições submersas contendo xilano, na temperatura de $30^{\circ} \mathrm{C}$, durante $72 \mathrm{~h}$.

A Figura 1 apresenta os Diagramas de Pareto para a AEC e AEX do fungo T. harzianum.
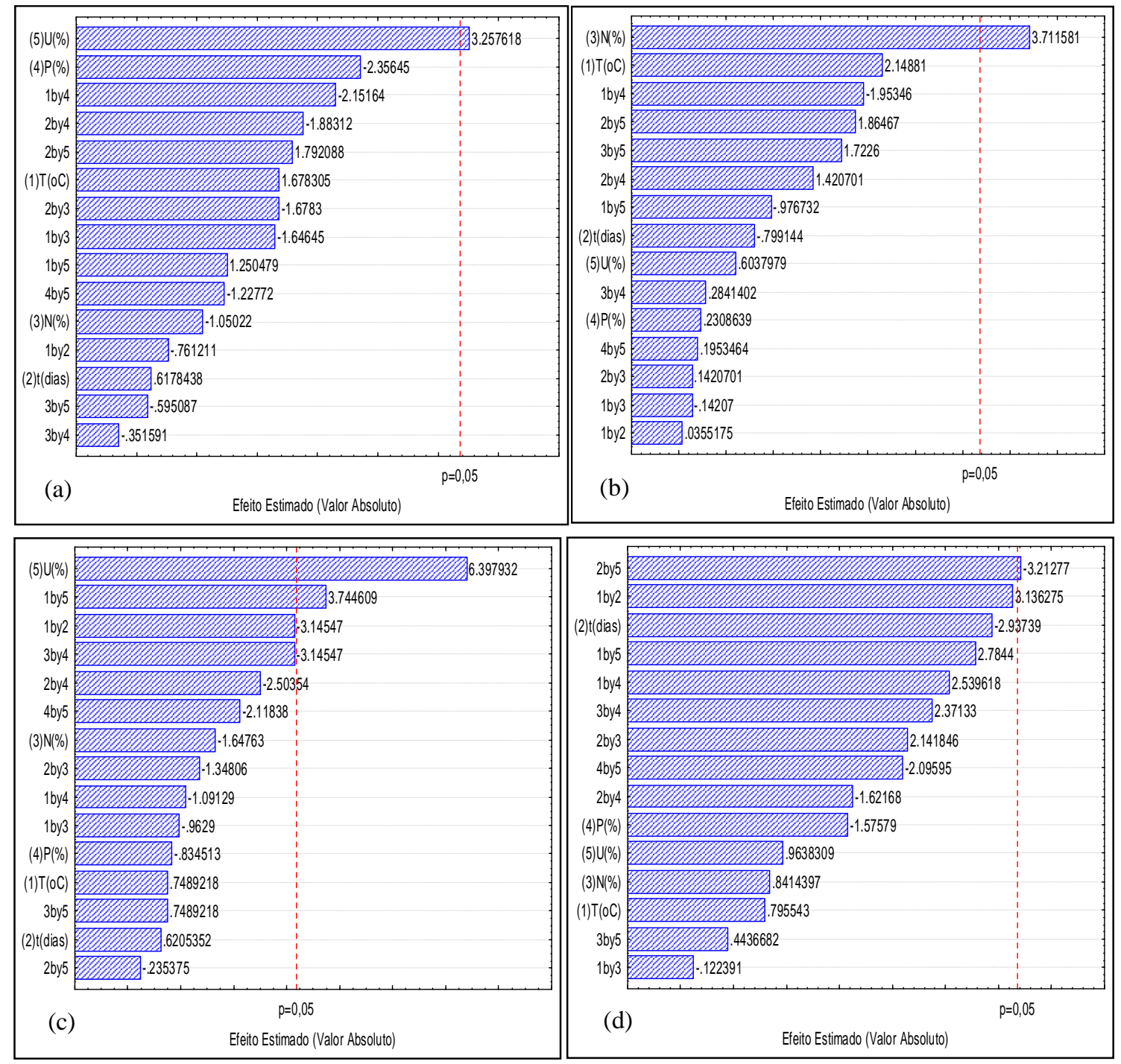

Figura 1 - Diagramas de Pareto para a atividade enzimática celulolítica do fungo Trichoderma harzianum cultivado no resíduo da macacaporanga, inoculado na forma de suspensão de esporos (a); e para a atividade enzimática xilanolítica do fungo Trichoderma harzianum cultivado no resíduo da macacaporanga, inoculado na forma de disco micelial (b), suspensão de esporos (c) e cultivado no resíduo de pau-rosa, inoculado na forma de disco micelial (d). 


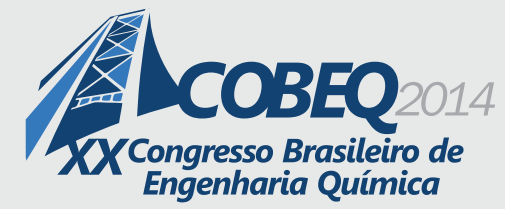

De acordo com a Figura 1a, somente a umidade foi estatisticamente significativa para o nível de confiança de $95 \%$, utilizando o inóculo na forma de SE e o resíduo de macacaporanga. Para os experimentos onde se utilizou o inóculo na forma de DM e os resíduos de macacaporanga e de paurosa, assim como o inóculo na forma de SE e o resíduo de pau-rosa, para a produção de celulase pelo fungo T. harzianum, nenhum dos efeitos mostrou-se estatisticamente significativo.

De acordo com a Figura $1 b$, somente a adição de nitrogênio foi estatisticamente significativa para o nível de confiança de $95 \%$ e influencia positivamente a produção da xilanase, utilizando o inóculo na forma de DM e o resíduo de macacaporanga. Na Figura 1c, nota-se que utilizando o inóculo na forma de SE e o resíduo de macacaporanga, assim como observado na Figura 1d (inóculo na forma de DM e o resíduo de pau-rosa), somente a umidade mostrou-se significativa e que a mesma influencia positivamente na produção da xilanase. Os efeitos da adição de nitrogênio e fósforo, temperatura e tempo de cultivo aparecem na sequência, e não apresentaram significância estatística.

Para os inóculos na forma de DM e de SE verifica-se que para a produção da xilanase no resíduo de pau-rosa nenhum dos efeitos (umidade, nitrogênio, tempo de cultivo, fósforo e temperatura) apresentou-se estatisticamente significativo para o nível de confiança de $95 \%$.

\section{CONCLUSÃO}

Os resíduos de Aniba, gerados a partir da hidrodestilação de galhos e folhas podem ser utilizados na FS para o cultivo do fungo T. harzianum, visando a obtenção das enzimas celulase e xilanase. Entretanto, os valores de atividade enzimática obtidos foram baixos, provavelmente devido à presença de linalol, composto com ação antifúngica, o qual deve ser eliminado do resíduo antes do bioprocesso. Na triagem das variáveis significativas para a produção de enzimas hidrolíticas, o melhor substrato para o cultivo de T. harzianum foi o resíduo de macacaporanga com $90 \%$ de umidade, sendo o inóculo na forma de SE o mais adequado para a produção de celulase e xilanase.

\section{AGRADECIMENTOS}

Os autores agradecem à FAPEAM, CNPq e CAPES pelo suporte financeiro, ao INPA e à EST/UEA pela infraestrutura concedida para a realização deste trabalho.

\section{REFERÊNCIAS}

FABER, K. Biotransformations in organic chemistry. $5^{\mathrm{a}}$ ed. Berlin: Springer, 2004.

INSTITUTO ADOLFO LUTZ. Normas Analíticas do Instituto Adolfo Lutz. 3. ed. São Paulo: O Instituto, 1985.

KAR, S.; MANDAL, A.; MOHAPATRA, P. K.; MONDAL, K. C.; PATI, B. R. Production of cellulose-free xilanase by Trichoderma reesei SAF3. Braz. J. Microbiol., v. 37, p. 462-464, 2006.

MACIEL, G. M. Desenvolvimento de bioprocesso para produção de xilanases por fermentação no estado sólido utilizando bagaço de cana de açúcar e farelo de soja. Dissertação de Mestrado. Programa de Pós-Graduação em Processos Biotecnológicos, UFP, Curitiba, 146p., 2006. 


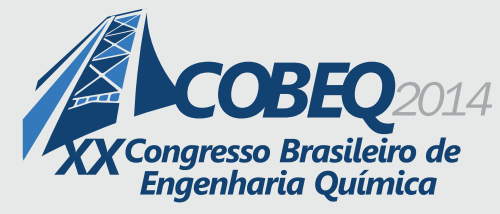

19 a 22 de outubro de 2014

Florianópolis/SC

MAIA, J. G. S.; ANDRADE, E. H. A.; COUTO, H. A. R.; SILVA, A. C. M. da; MARX, F.; HENKE, C. Plant sources of Amazon rosewood oil. Quim. Nova, v. 30, p. 1906-1910, 2007.

MAURYA, D. P.; SINGH, D.; PRATAP, D.; MAURYA, J. P. Optimization of solid state fermentation conditions for the production of cellulase by Trichoderma reesei. J. Environ. Biol., v. 33, p. 5-8, 2012.

MARQUES, C. A. Importância econômica da família Lauraceae Lindl. Floresta e Ambiente, v. 8, p. 195-206, 2001.

MELO, C. T.; MONTEIRO, A. P.; LEITE, C. P.; ARAÚJO, F. L.; LIMA, V. T.; BARBOSAFILHO, J. M.; FONTELES, F. M. M.; VASCONCELOS, S. M.; VIANA, B. G. S.; SOUSA, F. C. Anxiolyticlike effects of (O-methyl)-N-2,6-dihydroxybenzoyltyramine (apanese III) from Aniba riparia (Nees) Mez (Lauraceae) in mice. Biol. Pharm. Bull., v. 29, p. 451-454, 2006.

METZLER, D. E. Biochemistry, the chemical reactions of living cells. $2^{\mathrm{a}}$ ed., San Diego: Academic Press, 2001.

MILLER, G. L. Use of dinitrosalicilic acid reagent for determination of reducing sugar. Anal. Chem., v. 31, p. 426-428, 1959.

MUSSATTO, S. I.; FERNANDES, M.; MILAGRES, A. M. M. Enzimas: Poderosa ferramenta na indústria. Ciência Hoje, v. 41, p. 28-23, 2007.

PYBUS D. H.; SELL C. S. The chemistry of fragrances. Cambridge: The Royal Society of Chemistry, 1999.

REZENDE, M. I.; BARBOSA, A. M.; VASCONCELOS, A. F. D.; ENDO, A. S. Xylanase production by Trichoderma harzianum Rifai by solid state fermentation on sugarcane bagasse. Braz. J. Microbiol., v. 33, p. 67-72, 2002.

RODRIGUES, I. M.; IEMMA, A. F. Planejamento de experimentos e otimização de processos, Campinas: Casa do Pão, 2005.

RUEGGER, M. J. S.; TAUK-TORNISIELO, S. M. Atividade da celulase de fungos isolados do solo da Estação Ecológica de Juréia-Itatins, São Paulo, Brasil. Rev. Bras. Bot., v. 27, p. 205-211, 2004.

SILVA, L. A. O.; CARMONA, E. C. Production and characterization of cellulase-free xylanase from Trichoderma inhamatum. Appl. Biochem. Biotec., v. 150, p. 117-125, 2008.

SINGHANIA, R. R.; PATEL, A. K.; SOCCOL, C. R.; PANDEY, A. Recent advances in solidstate fermentation. Biochem. Eng. J., v. 44, p. 13-18, 2009.

SOUZA, T. D.; VINHA, D. M. N. F.; BISPO, R. S. A.; BOM, S. D. P. E, COELHO, R. R. R.; NASCIMENTO, D. P. R. Produção de xilanases pelo fungo Aspergillus fumigatus FBSPE-05, em fermentação semi-sólida (FSS), utilizando diferentes resíduos agroindustriais. XVII Simpósio Nacional de Bioprocessos. Natal/RN, Livro de resumos, 2009. 